A N N A L E S Annales de Bretagne et des Pays de l'Ouest

Anjou. Maine. Poitou-Charente. Touraine

111-3 | 2004

Alcuin de York à Tours

\title{
Les motifs épistolaires dans la correspondance d'Alcuin
}

\section{Christiane Veyrard-Cosme}

\section{(2) OpenEdition}

\section{Journals}

Édition électronique

URL : http://journals.openedition.org/abpo/1238

DOI : $10.4000 / a b p o .1238$

ISBN : 978-2-7535-1495-9

ISSN : 2108-6443

Éditeur

Presses universitaires de Rennes

Édition imprimée

Date de publication : 20 septembre 2004

Pagination : 193-205

ISBN : 978-2-7535-0053-2

ISSN : 0399-0826

\section{Référence électronique}

Christiane Veyrard-Cosme, «Les motifs épistolaires dans la correspondance d'Alcuin », Annales de Bretagne et des Pays de l'Ouest [En ligne], 111-3| 2004, mis en ligne le 20 septembre 2006, consulté le 20 avril 2019. URL : http://journals.openedition.org/abpo/1238 ; DOI : 10.4000/abpo.1238 


\title{
Les motifs épistolaires dans la correspondance d'Alcuin
}

\author{
Christiane VEYRARD-COSME \\ Maître de conférences de Langue et littérature latines, Université de Paris 3 \\ Laboratoire de médiévistique occidentale de Paris (LAMOP, UMR 8589)
}

La lettre, souvent utilisée comme document, autorise, en tant que discours, une approche pragmatique au sens linguistique du terme, étudiant l'énonciation afin d'en appréhender le mode de communication.

En offrant un aperçu des motifs épistolaires dans la correspondance d'Alcuin, nous aimerions voir comment la lettre se fait emblème et substitut d'une action; nous évoquerons d'abord le rôle joué par les motifs épistolaires dans la définition de la lettre alcuinienne, puis dans l'émergence d'une identité de l'épistolier, avant de voir, enfin, l'importance des motifs épistolaires pauliniens dans la transformation de la lettre en espace eschatologique.

\section{Motifs épistolaires et nature de la lettre}

\section{Le motif de l'absentia corporis}

Si la pratique épistolaire emprunte à la rhétorique nombre de ses armes, les lettres d'Alcuin, déterminées socialement et historiquement, élaborent pourtant une topique particulière, qui nourrit une poétique particulière. Les motifs épistolaires, chez Alcuin, peuvent être liés à l'échange et à la matérialité de l'objet lettre ou contingents, dépendant du type de lettre envoyée, et souvent empruntés à des textes littéraires, voire à d'autres correspondances. Certains motifs viennent aussi de textes scripturaires, thématisés par Alcuin et utilisés avec une fréquence haute, si bien que dans ce cas le motif naît de la répétition. Mais répétition ne signifie pas répétition à l'identique, car le motif se transforme en fonction des éléments du texte qui le reçoit et des éléments avec lesquels il se combine, au point de devenir parfois un leurre.

L'antique référence à la lettre-conversation entre absents, présente chez Alcuin via des citations de Jérôme reprenant Cicéron, occulte ainsi la nature du discours épistolaire, qui requiert un destinataire et sa distanciation, 
condition de l'émergence d'une écriture évoquant l'épistolier plus qu'un destinataire souvent idéalisé, ou qu'un univers sur lequel la parole aurait le pouvoir d'agir de manière immédiate ${ }^{1}$.

Réexaminons le motif alcuinien de l'absentia corporis, souvent relevé par les chercheurs, dans une perspective toutefois différente de la nôtre ici, motif partagé par les correspondances amoureuses et amicales. Si la lettre 28 d'Alcuin à Paulin d'Aquilée livre cet adage ${ }^{2}$ : « l'absence physique ne doit pas séparer ceux qui s'aiment ", les lettres 26 et 35, adressées à Riculf, avancent le motif de cette manière :

"La joie que me donne la douceur de l'amour est à la mesure de la souffrance que me donnent éloignement et absence. Je n'aurais pas voulu voir si longtemps séparés l'un de l'autre ceux qu'unissent les si doux liens d'une même charité. Que puis-je faire, sinon suivre de mes larmes, et jusqu'à son retour, l'ami dont mon âme souhaite tant la présence ${ }^{3}$ ?"

"Il y a bien trop longtemps, me semble-t-il, que je n'ai vu ta Dilection en personne, que je n'ai entendu tes propos. La joie qu'éprouve mon âme à ta présence est à la mesure de la tristesse que lui donne ton absence ${ }^{4}$. "

Si la structure dialogique est donnée par l'alternance des pronoms personnels ou adjectifs possessifs de la première et de la deuxième personne du singulier, la composition cyclique montre souvent, comme dans l'extrait suivant ${ }^{5}$, la prédominance du taedium, dégoût et lassitude provoqués par l'absence $^{6}$ :

"L'absence de ta personne pèse de tout son poids sur mon âme. Que la vie était douce quand nous étions assis, au calme, parmi les écritoires du savoir et les livres nombreux! Tout m'est poids et tristesse dans l'attente et le désir où je suis de vous voir."

\section{Alcuin, le « syndrome d'Habacuc » et la critique contemporaine}

À ce motif se rattache ce que nous serions tentée d'appeler " le syndrome d'Habacuc ", qui se déploie dans plusieurs lettres exploitant un pas-

1. Sur ces points cf. C. VeyraRd-CoSmE, "Saint Jérôme..." ".

2. Alc. Ep. 28, p. 70 : Absentia corporis non oportet dilectionem diuidere. Nous citerons notre traduction des Lettres d'Alcuin (à paraître, avec commentaire thématique, en Sources Chrétiennes).

3. Alc. Ep. 26, p. 67 : Sed quantum gaudeo in amoris dulcedine, tantum doleo in absentiae longinquitate. Noluissem tanto tempore ab inuicem separari, quos eiusdem caritatis dulcissima colligant uincula. Quid faciam nisi lacrimis sequar amicum donec reueniat quem animus optat habere praesentem.

4. Alc. Ep. 35, p. 77 : Nimium mihi longum videtur tempus, quod tuae dilectionis faciem non uidi, uerba non audiui. Et quantum ex praesentia tui gaudebat animus, tantum in absentia contristatur.

5. Alc. Ep. 281, à un élève, p. 439 : Tedet animus meus multum pro absentia tui. O quam dulcis uita fuit dum sedebamus quieti inter sapientis scrinia, inter librorum copias [...] Ego uero tediosus et tristis desiderabilem uestrae faciei praestolabor aspectum [...].

6. Cf. également Alc. Ep. 49 à Ricbod, p. 93 : fateor, mea mentis dilectio tui tedet absentiam. 
sage de la lettre III de Jérôme à Rufin ${ }^{7}$. Cette reprise s'observe dans la lettre 10, envoyée à Arn de Salzbourg ${ }^{8}$ :

"Oh, si seulement m'était soudainement accordée la translation d'Habacuc, avec quelle rapidité je me précipiterais pour vous prendre dans mes bras, Père, et avec quelle force, de ma bouche, j'embrasserais non seulement yeux, oreilles, visage mais aussi mains et doigts de pieds un par un, maintes et maintes fois! "

On l'observe également dans la lettre 193, destinée à Arn ${ }^{9}$, dans la lettre 229 , à Charles ${ }^{10}$, et la lettre 83 , à Pierre de Milan ${ }^{11}$. Toutefois, c'est la lettre 10 qui fait l'objet de la plupart des analyses; Marta Cristiani en livre cette interprétation ${ }^{12}:$ " La rhétorique de l'hommage, un hommage chargé - il est vrai d'affectivité, est probablement la clé de ce texte. " Elle voit dans le lyrisme alcuinien, qu'elle nomme " affectivité profonde ", un "signe de raffinement intellectuel " en le reliant à un souci pédagogique ${ }^{13}$ :

"C'est la parole écrite, c'est l'art raffiné de la correspondance qui devient l'instrument pédagogique fondamental, quand la communication directe, l'échange personnel des sentiments et des savoirs est devenu impossible."

Wolfgang Edelstein, lui, note dans ces passages ce qu'il appelle " die affektive Intensität ", dont le haut degré est l'originalité d'Alcuin ${ }^{14}$.

Pour Adele Fiske, ces lignes appellent ce commentaire ${ }^{15}$ : " Les expressions de tendresse d'Alcuin, ne sont, au demeurant, pas originales dans leur forme, ni exemptes d'exagérations rhétoriques; néanmoins, elles sont sincères et personnelles. " Donald A. Bullough, enfin, dans un chapitre inti-

7. Lettre III (t. 1, p. 10-11) : O si mihi nunc Dominus Iesus Christus uel Philippi ad eunuchum uel Ambacum ad Danihelum translationem repente concederet, quam ego nunc arte tua stringerem colla conplexibus, quam illud os, quod mecum uel errauit aliquando uel sapuit, inpressis figerem labiis! Verum, quia non mereor et inualidum etiam cum sanum est corpusculum crebri fregere morbi, has mei uicarias et tibi obuias mitto, quae te copula amoris innexum ad me usque perducant.

8. Alc. Ep. 10, p. 36 : O, si mihi translatio Abacuc esset subito concessa, quam citatis manibus ruerem in amplexus paternitatis uestrae, et quam compressis labris non solum oculos aures et os, sed etiam manuum uel pedum singulos digitorum articulos, non semel, sed multoties oscularer.

9. Alc. Ep. 193, p. 319 : O si mihi translatio Abacuc esset concessa ad te, quam tenacibus tua colla strinxissem, o dulcissimi fili, amplexibus.

10. Alc. Ep. 229, p. 373 : O Domine Iesu, quare non das mihi pennas aquilae? Quare non translationem Abacuc prophetae una die uel etiam hora concedis? ut amplecter et osculer uestigia illius carissimi mei et super omne quod in hoc mundo amari potest, dulcissimi oculos uideam clarissimos, uerba audiam iocundissima.

11. Alc. Ep. 83, p. 126 : O si pennas aquilae habuissem, ut altitudines Alpium velocior Euro transuolare ualuissem, quam cito ante paternos stetissem pedes, ut refrigerarem ex paterna uisione pectoris mei ardorem.

12. M. CRISTIANI, "Le vocabulaire de l'enseignement... ", p. 18.

13. Ibid., p. 16-17.

14. W. EdELSTEIN, Eruditio..., p. 46.

15. A. FISKE, "Alcuin and mystical friendship ", p. 574 : "Alcuin's expressions of tenderness are not, indeed, original in form nor without rhetorical exaggerations; yet they are sincere and personal ". 
tulé "Amicitia and Sexual Orientation ", commente ainsi la lettre $10{ }^{16}$ : "L'amitié est ici assortie d'un langage haut en couleur, tout d'émotion, exprimé par un vocabulaire du contact physique qu'il est presque impossible de ne pas décrire comme sensuel ou érotique. " Et tout en rappelant aux lecteurs que, selon les époques, l'expressivité peut emprunter des modalités différentes, et en les mettant en garde contre une interprétation hâtive qui verrait dans ces textes l'expression d'une homosexualité, Donald A. Bullough déclare ${ }^{17}$ : «Même ceux qui, raisonnablement, sont les tenants d'une interprétation métaphorique ou allégorique du langage de l'amitié d'Alcuin ont encore à expliquer comment il en est venu à l'adopter. "

\section{Motifs épistolaires et fiction narrative}

Or, le texte de Jérôme, devenu motif, est utilisé par Alcuin dans des lettres qui ne sont pas exclusivement adressées à un unique correspondant, puisqu'elles concernent aussi bien Arn que Pierre de Milan ou Charlemagne; de plus, ce motif repose sur l'irréel, brèche sur un univers virtuel dans un discours qui lui-même se fonde sur une reconstitution de faits ; cet irréel, méthode épistolaire chère à Cicéron, comme l'a montré Florence Oliver ${ }^{18}$, et à Jérôme, instaure une coupure avec la réalité, non seulement par sa modalité, mais aussi par les éléments transmis. Le syndrome d'Habacuc ne peut être reflet d'une réalité. Bien mieux, absence et séparation spatio-temporelle sont des motifs si puissamment thématisés qu'ils inscrivent dans la lettre alcuinienne la situation énonciative de séparation corporelle. La lettre est absence faite corps, avant d'être présence à l'autre.

Une autre expression d'Alcuin, dans la lettre 83, à Pierre de Milan ${ }^{19}$ - Concessum est humano generi pectoris arcana cartis mandare - montre enfin que, dans ces lettres qui vont d'un cœur à l'autre, "s'écrire " devient réflexif ${ }^{20}$.

La lettre alcuinienne véhicule une image du scripteur adressée à un autre, privilégié, qui est aussi reconstruit par l'échange épistolaire. Il conviendrait, pour reprendre les distinctions opérées par Christine Servais $^{21}$, de recourir aux notions de récepteur pour désigner celui qui reçoit dans la réalité cette lettre, d'interscripteur pour désigner cet autre

16. D.A. Bullough, Alcuin : achievement and reputation..., chapitre " Amicitia and Sexual Orientation ", p. 110-117 (citation, p. 113) : "Amicitia is here associated with a highlycoloured, emotional language, expressed in the vocabulary of physical contact and almost impossible not to describe as sensual or erotic" .

17. Ibid., p. 114 : " Even those, who, reasonably, hold to a metaphorical or allegorical interpretation of Alcuin's language of friendship have still to explain how he came to adopt it ".

18. F. OLIVER, " Le temps virtuel..." .

19. Alc. Ep. 83, p. 126.

20. Cf. sur ce point, B. Diaz, L'épistolaire..., p. 88.

21. Ch. Servais, " Le double jeu... ", p. 116. 
auquel Alcuin écrit, cet alter ego idéalisé, auquel, comme on le sait, il attribue des surnoms - parfois plusieurs pour un même récepteur ${ }^{22}$-, en plus de la notion de destinataire qui peut renvoyer aussi bien au lectorat élargi de l'époque qu'à celui qui lit, bien plus tard, une correspondance qui ne lui était point adressée. Qui plus est, le je qui écrit se projette vers un destinataire - mandare pectoris arcana - par définition si distant que la subjectivité à l'œuvre a tout de la fiction narrative à la première personne ${ }^{23}$.

La lettre alcuinienne n'est donc point, à notre sens, document brut ou réalité donnée en transparence. Elle offre plutôt une construction de soi et du monde, qui, sous la surface lisse d'un texte écrit à la première personne, laisse affleurer, grâce aux motifs, d'autres figures.

\section{Les motifs épistolaires ou la question de l'identité}

\section{Un épistolier porte-voix}

Qui parle sous le je de l'épistolier Alcuin? Bien d'autres épistoliers, mais surtout Jérôme auquel, comme le supposait Mary Alberi et comme nous avons eu également l'occasion de le montrer, Alcuin emprunte un autoportrait en Flaccus, dont le point de départ se trouve dans la lettre VI de Jérôme ${ }^{24}$. Certains rapprochements troublants se font d'ailleurs, à notre sens, entre surnoms alcuiniens et hiéronymiens : chez Jérôme, Timothée, surnom réservé à ses correspondants amis, désigne ainsi Népotien dans la lettre LX et, dans la lettre LIII - dont l'adresse est ad Paulinum presbyterum - devient celui de Paulin : le Stridonien, acceptant d'être pour Paulin un compagnon dans l'étude sacrée, se compare à Paul instruisant Timothée et voit en Paulin un autre Timothée. Or, c'est du même nom de Timothée que le clerc Alcuin aime à surnommer son ami... Paulin d'Aquilée! Bien mieux, dans la lettre CVII à Laeta, sur l'éducation des filles, Jérôme établit un parallèle entre sa personne et celle d'un dénommé Albinus, présenté dans ces lignes comme un homme âgé instruisant une uirgo Christi ${ }^{25}$. Jérôme, reprenant parfois mot pour mot sa présentation d'Albinus, promet donc à Laeta d'instruire, à l'exemple de cet homme, sa fille Paula ${ }^{26}$. Quand on sait que l'épistolier Alcuin aime à se présenter comme Flaccus Albinus, de telles " correspondances " ont de quoi étonner...

22. Alc. Ep. 281, p. 439 : pater filio, pacificus peregrino, magister discipulo, socius socio peregrinationis sempiternam salutem. Sur les surnoms, M. GARRISON, « The Social World of Alcuin...".

23. Sur l'auto-fiction, l'ouvrage de P. GASPARINI, Est-il je?...

24. Lettre VI, p. 20 : Verum omnem culpam praesens uerbositas excusabit. Nam, ut ait Flaccus in satura : "Omnibus hoc uitium est cantoribus, inter amicos rogati ut numquam cantent, iniussi numquam desistant, ita te deinceps fascibus obruam litterarum, ut e contrario incipias rogare ne scribam. "

25. Lettre CVII, p. 145 : Quis hoc crederet ut Albini pontificis neptis de repromissione matris nasceretur, ut praesente et gaudente auo adhuc lingua balbuttiens " alleluia "resonaret et uirginem Christi in suo gremio nutriret et senex?

26. Ibid., p. 158 : Si Paulam miseris, et magistrum me et nutricium spondeo [...] balbutientia senex uerba formabo [...] ancillam et sponsam Christi erudiam... 
Le motif épistolaire pose à son tour la question de la polyphonie d'un texte. Prenons la lettre 227 d'Alcuin ${ }^{27}$ :

"Que tu entres ou sortes, veilles ou dormes, la douceur de l'amour paternel ne cesse de te suivre; où que tu ailles même, puisse son souvenir aller et demeurer pour toujours à tes côtés, car à ton départ comme à ton retour, sa charité pour toi ne cesse de te suivre pas à pas. Sache-le, et sois en sûr, une mère n'attend pas dans son anxiété l'arrivée de son fils parti en voyage, avec plus d'ardeur que mon cœur n'éprouve le désir de revoir ton visage. "

Elle porte l'écho des lettres de Pline à Calpurnie, ou Julius Servianus, et, sous le thème de la mère anxieuse, reprend le motif de Jérôme dans sa lettre III à Rufin ${ }^{28}$. Ce sont là des rapprochements que ne connaît pas l'éditeur des MGH, mais qui témoignent des échos possibles d'une correspondance à d'autres correspondances ${ }^{29}$.

Alcuin épistolier avance ses lettres comme un chant plaintif - suivant en cela Jérôme qui, dans sa lettre VI, définit, par ricochet, l'épistolier comme cantor $^{30}$ et appelle certaines lettres cantilenae ${ }^{31}$ - comme le montre l'adresse de la lettre 59 à Arn, qui définit, en tant qu'adresse, un contrat de lecture et une clé d'interprétation du contenu de la missive ${ }^{32}$ :

"Au père de sa très douce affection, à l'aigle, à l'évêque, le cygne de l'audelà des mers adresse le salut perpétuel sur les ailes de la sainte charité. »

Cignus, loin d'être un simple surnom animal, vient de l'autoportrait de Jérôme, qui dans sa lettre LII à Népotien, se compare aux grandes figures de poètes donnant leur chant du cygne ${ }^{33}$.

Il vient aussi du passage de la lettre LX à Héliodore, éloge funèbre du même Népotien, évoqué ainsi par Jérôme : Ubi est cygneo canore vox dulcior?

Ce motif, chez Alcuin, entre en combinaison avec deux autres motifs hiéronymiens, celui de la lettre transmarina, où la mer, réalité de l'échange, est aussi métaphore des dangers possibles ${ }^{34}$ et celui de la caritas ailée.

27. Alc. Ep. 227, à Arn séjournant à Rome, p. 371 : Ingredientem et egredientem, uigilantem, dormientem semper te sequitur dulcedo paternae dilectionis; immo ubicumque uadas, tecum eat, tecum aeterna maneat memoria, quia te euntem et redeuntem illius semper consequitur caritatis affectus. Scias certissime quod nec sic anxia peregrinantis mater filii spectat aduentum, quanto mei cordis ardentissima uoluntas tuae faciei praestolatur aspectum.

28. Lettre III, p. 12 : Credas mihi uelim, frater, non sic tempestate iactatus portum nauta prospectat... non sic curuo adsidens litori anxia filium mater expectat.

29. Cf. aussi le motif de la blessure de charité : Alc. Ep. 59, 159 et 172 d'Alcuin avec les lettres LXXV, LXV, LX et LII de Jérôme développant Cant 2, 5 (uulnerata caritatis ego).

30. Lettre VI, p. 20.

31. Lettre CIX, p. 206.

32. Alc. Ep. 59, p. 102 : Dulcissimae dilectionis patri aquilae antistiti transmarinus cignus in sanctae caritatis pennis perpetuam salutem.

33. Cf. lettre LII, p. 175 : ad poetas uenio, Homerum, Hesiodeum, Simonidem, Stesichorum, qui grandes natu cygneum nescio quid et solito dulcius uicina morte cecinerunt.

34. Cf. lettre LII, 1 à Népotien (litterae transmarinae) ou lettre LX, 11 à Héliodore, t. 3, p. 100 : Quotiens ille transmarinis epistulis deprecatus est ut aliquid ad se scriberem! 
L'importance, au plan sémiotique, du motif connexe de la lettre ailée que portent de nombreuses lettres alcuiniennes, écrites à la plume par un cygne-épistolier dont le chant s'élance parfois vers l'aigle Arn ${ }^{35}$, montre que la lettre est un énoncé " volage ", et dans l'image qu'en donne l'épistolier, et dans sa réception, puisque, de lecteur en lecteur, cet énoncé à la première personne se laisse apprivoiser ${ }^{36}$.

Porte-voix, l'épistolier Alcuin avance aussi une identité faite d'altérité et une peinture du monde carolingien aux couleurs patristiques.

\section{Une identité faite d'altérité}

Alcuin puise dans les lettres CV, LX et LII de Jérôme le motif du vétéran : ce motif guerrier lui permet de faire circuler le texte à différents niveaux, celui de Charles par exemple dans l'Ep. 240 qui comporte un distique en l'honneur de la dextera David à laquelle le Dieu tout-puissant a accordé la victoire ${ }^{37}$, celui de Dieu, le véritable Seigneur des armées, et celui du miles Christi qu'est l'épistolier.

Prenons la lettre 240 : Ecce Flaccus effeto corpore militaris cingulo laboris deposito... Deo soli quieta pace seruire toto elegit desiderio. L'éditeur des MGH voit dans effeto corpore une référence à Boèce. Toutefois, l'examen de certaines adresses de lettres, comme celle de la lettre 149 (Flaccus, ueteranus miles), ou celle de la lettre 155 (miles ueteranus Flaccus), ou l'étude de la lettre 145 menée par Mary Alberi, qui, la première, souligna les points de convergence de la lettre 145 avec la lettre CV de Jérôme, montrent les liens de ce thème avec la lettre $\mathrm{CV}$ de Jérôme à Augustin ${ }^{38}$ : Ego quondam miles, nunc ueteranus, et tuas et aliorum debeo laudare uictorias, non ipse rursus effeto corpore dimicare ${ }^{39}$, ou même avec la lettre LX à Héliodore, qui parle du cingulum et évoque le sort du vétéran, balteo posito ${ }^{40}$.

On connaît aussi par la lettre 121 adressée à Charles l'intérêt que porte Alcuin à la lettre LII de Jérôme ${ }^{41}$ :

" J'ai pour me réconforter, moi dont le corps est brisé, cette remarque de saint Jérôme qui dit dans sa lettre à Népotien : Chez les vieillards presque

35. Cf. Alc. Ep. 264, p. 421; Alc. Ep. 78, p. 119, à Ricbod : Et uolat saepius carta, caritatis alis pennata A comparer avec Jérôme, lettre IV, p. 16 : Ego ille tardissimus, quoniam intolerabilis languor, pinnatis ut aiunt pedibus charta caritatis et uoto te salutauerim et iam conplexus sim! gratulor itaque tibi et nascentem amicitiam ut Dominus foederare dignetur precor.

36. B. Diaz, L'épistolaire ..., parle d'énoncé migrateur, p. 246.

37. Discingat chartam mitis, rogo, dextera David Victricem faciat quam Deus almipotens. 38. M. ALBERI, « Jerome... ", pour les premiers rapprochements, et lettre CV, 3 (t. 5), p. 101. 39. Alc. Ep. 240, p. 385.

40. Lettre IX (t. 3), p. 97.

41. Alc. Ep. 121 : Mihique, fracto corpore, solatio est sententia sancti Hieronimi, qui ait in epistola ad Nepotianum : "Omnes pene uirtutes corporis mutantur in senibus et, crescente sola sapientia, decrescunt cetera. ”Et post paululum : "Senectus uero eorum, qui adulescentiam saum honestis artibus instruxerunt et in lege Domini meditati sunt die ac nocte, aetate fit doctior, usu tritior, processu temporis sapientior; et ueterum studiorum dulcissimos fructus metet. » 
toutes les facultés du corps s'altèrent; seule s'accroît la sagesse mais toutes les autres décroissent; Puis peu après la vieillesse des hommes dont la jeunesse s'est instruite des belles-lettres et qui ont ensuite médité sur la loi de Dieu jour et nuit, cette vieillesse devient, grâce à Dieu, plus docte, grâce à l'usage de la vie plus expérimentée et plus sage, à mesure que s'avance le temps; enfin, de ses études d'autrefois elle récoltera des fruits très savoureux."

Cette lettre LII de Jérôme nourrit la réflexion d'Alcuin sur le rôle de l'évêque, fournit des prescriptions sur les vêtements, le vin, le jeûne, le miles Christi; l'éditeur des MGH l'ignore, mais elle est bien la source du célèbre passage de la lettre 290 demandant de ne pas porter les ossements des saints sur soi ${ }^{42}$. Et c'est encore cette lettre LII qui transparaît dans la lettre 136 où Alcuin évoque la parole des prêtres ${ }^{43}$.

Or, cette lettre LII, fondamentale pour le miroir sacerdotal chez Alcuin, est aussi celle qui parle des cygnes; le thème du vétéran offre donc à Alcuin la possibilité d'amorcer, avec le thème du soir de la vie, les éléments d'un testament spirituel tout en faisant de sa vie une leçon, destinée au lecteur, qui en suivra l'exemple ou en rejettera les erreurs. C'est ainsi que, s'appuyant sur le célèbre " catalogue des circonstances de vie " paulinien que présente 2 Co 11, notamment le verset 25 (ter naufragium feci), l'épistolier

42. Jérôme, lettre LII, 13 - Nolo te dilatare fimbrias et ostentui habere pulakteria et conscientia repugnante pharisaeica ambitione circumdari. Melius est haec in corde portare quam in corpore, deum habere fautorem, non aspectus hominum - et Alc. Ep. 290, p. 448 : Sed melius est in corde sanctorum imitare exempla quam in sacculis portare ossa; euangelicas habere scriptas ammonitiones in mente magis, quam pittaciolis exaratas in collo circumferre. Haec est pharisaica superstitio, quibus ipsa ueritas improperauit philacteria sua.

43. Alc. Ep. 136, p. 209 (cf. la traduction, infra p. 465) : Audio etiam per ecclesias Christi quandam consuetudinem non satis laudabilem quam uestra prudentissima auctoritas faciele emendare potest. Si tamen uera est opinio, et non magis falsa excusatio ut quod facere non uolunt presbyteri suis iniciant episcopis. Nam dicunt ab episcopis interdictum esse presbyteris et diaconibus praedicare in ecclesiis; dum in apocalypsi legatur : "Spiritus et sponsa dicunt : Veni et qui audiat dicat : veni. Qui sitit ueniat; qui uult, accipiat aquam uitae gratis ». Nec non et apostolus : "Quod si alii reuelatum fuerit sedenti, prior taceat; potestis enim per singulos prophetare, id est docere; item ad Timotheum : Qui bene praesunt presbyteri, duplici honore digni habeantur; maxime qui laborant in uerbo et doctrina Dei. "Quin magis legant et intellegant ab initio nascentis ecclesiae quanti et quam mirabiles ex diuerso clericorum ordine per totam mundi latitudinem fuere praedicatores etiam et apostolica in diuersas partes transmissi auctoritate Et desinant speciale habere quod ad maius animarum lucrum plurimorum poterit esse. Jérôme, lettre LII (t. 2, p. 182) : Pessimae consuetudinis est in quibusdam ecclesiis tacere presbyteros et praesentibus episcopis non loqui, quasi aut inuideant aut non dignentur audire. Et si alii, inquit Paulus apostolus, "fuerit reuelatum sedenti prior taceat. Potestis enim per singulos prophetare ut omnes discant et omnes consolentur. Et spiritus prophetarum prophetis subiectus est; non enim est dissensionis deus, sed pacis ». Gloria patris est filius sapiens : gaudeat episcopus iudicio suo cum tales Christo elegerit sacerdotes. Puis Alcuin reprend : "Dicit enim beatus Hieronimus in epistola ad Nepotianum presbyterum, ubi eum de praedicationis officio instituit » et il cite LII, 7 et le début de 8 (p. 182-183). On notera qu'Alcuin, dans cette même lettre, mentionne sa rusticitas à la fin de ce passage, et dans l'adresse se nomme Flaccus, en deux mentions hiéronymiennes. 
se dit naufragus ${ }^{44}$ ou met en avant son infirmitas ${ }^{45}$. En accord avec 2 Co 12 , 10 ("Voilà pourquoi je suis content des faiblesses, des outrages, des nécessités, des persécutions, des angoisses pour le Christ, car lorsque je suis faible, c'est alors que je suis fort "), chez Alcuin, les malheurs des peuples anglo-saxons, les difficultés personnelles sont vues comme actualisation des souffrances christiques et lient destinataires et destinateurs dans l'Esprit, donnant à la faiblesse de proclamer le Christ ressuscité et la puissance divine.

La lettre alcuinienne, à la différence de l'autobiographie qui présuppose une reconstruction rétrospective unifiée, propose donc des bribes de fictions de soi dont certaines sont tirées vers l'exemplarité. Lieu de mémoire, où s'entremêlent les voix du passé, elle montre enfin, dans l'utilisation de motifs scripturaires à l'origine, une appréhension particulière des catégories de l'espace et du temps.

\section{Du motif paulinien \\ à la lettre alcuinienne comme espace eschatologique}

Les lettres d'Alcuin avancent 149 citations directes pauliniennes, comme nous avons eu l'occasion de le montrer récemment. Certes, citation directe et motif épistolaire ne sont pas toujours réductibles l'un à l'autre, mais lorsqu'une citation, de directe, c'est-à-dire insérée dans le corps de la lettre, se fait élément dérivé et fournit au développement vocabulaire et métaphore, elle excède son rôle traditionnel d'autorité, surtout si elle donne naissance à des développements récurrents susceptibles d'être mis en série dans un grand nombre de lettres.

44. Alc. Ep. 187, p. 314 : Ego uero, saeculi tempestatibus turbatus, casso multis in locis labore desudaui, sed modo quasi naufragus, Deo miserante, ad portum deiectus quietis, in quo me fessum et uulneratum iacentem deprecor sanctarum assiduitate prationum uestrae unanimitatis auxiliari.

45. Alc. Ep. 158, adresse, ou Alc. Ep. 146 à Arn, p. 236. Cf. aussi Alc. Ep. 114, p. 169, à comparer avec la lettre III de Jérôme, p. 14 : Fessa ieiuniis membra morbo grauante concutiet sed apostoli repercutietur eloquio : "quanto infirmor, tunc fortior sum et uirtus in infirmitate perficitur ». Minabitur mortem sed audiet : " cupio dissolui et esse cum Christo ».

46. Cf. 1 Th, Ph, Rm, 2 Co.

47. Alc. Ep. 143 de 798, p. 224 : Benedictus sit Deus pater omnipotens, qui te creauit et honorauit. Et benedictus sit dominus noster Iesus Christus filius Dei uiui, qui te redemit et elegit. Et benedictus sit Spiritus paraclitus, qui te inluminauit et dilatauit cor tuum in omni sapientiae et scientiae claritate, dilectissime Dauid et dulcissime domne [...] Et benedicta sit potestas et regnum tuum et filii tui et filii filiorum tuorum usque in generationes saeculi sempiternas; et ueniat super te et super tuam generationem benedictio sanctorum in die domini nostri Iesu Christi. Cuius sanctissima uoluntas semper uigeat floreat et crescat in corde tuo, clarissime ecclesiae Christi rector et defensor. Aussi Alc. Ep. 229, p. 372, ou Alc. Ep. 210, p. 350. 


\section{Lettres alcuiniennes et berakha paulinienne}

Les bénédictions qui nourrissent le formulaire paulinien ${ }^{46}$ transparaissent dans certaines lettres alcuiniennes ${ }^{47}$. Or, la berakha est louange qui allie bénédiction - chanter les grandeurs de Dieu - et action de grâces - au sens psalmique, qui signale les actions divines pour en faire mémoire par le récit -; d'un point de vue didactique, elle amorce les éléments que le protagoniste de l'échange épistolaire est appelé à mettre en pratique. Sur le plan parénétique, elle sert l'exhortation. Sur le plan de l'énonciation, elle conforte la prise de parole du scripteur qui délivre ces prescriptions et le campe du même coup en praedicator paulinien. Dans un univers où " dire c'est faire ", ce motif reflète donc l'auto-constitution de l'épistolier en prédicateur et permet à Alcuin d'orienter son lecteur vers une nouvelle appréhension du temps.

\section{La question du temps chez Alcuin épistolier}

Si Donald A. Bullough a souligné l'importance dans l'œuvre d'Alcuin du thème du Royaume des Cieux, ce thème n'a, à notre connaissance, fait l'objet d'aucune réflexion concernant la correspondance. Or, le temps, catégorie essentielle de l'épistolaire, est une notion propre à beaucoup de lettres alcuiniennes : l'écriture épistolaire d'Alcuin s'articule entre passé et futur qui dit l'espérance, l'optata praesentia, comme le montre la lettre 59 à $\mathrm{Arn}^{48}$ :

" Au père de sa très douce affection, à l'aigle, à l'évêque, le cygne de l'audelà des mers adresse le salut perpétuel sur les ailes de la sainte charité.

Attendre, espérer, appeler de tous ses vœux... Mais voici que celui que j'ai attendu n'est pas venu. Celui que j'ai espéré, je ne l'ai pas vu; que j'appelais de tous mes vœux, je ne l'accueillais point. Vaine, l'attente! Tari, l'espoir! Ah, si seulement il y avait un espoir de présence, la joie serait à son comble... Mais, hélas, en lieu et place de l'allégresse attendue, c'est la tristesse qui s'insinue, sans crier gare.

Que feras-tu, mon cœur blessé de charité? Est-il quelque soulagement à la mesure d'une si blessure si profonde, ou quelque baume? Rejettes-tu

\footnotetext{
48. Alc. Ep. 59, p. 102-103 : Dulcissimae dilectionis patri aquilae antistiti transmarinus cignus in sanctae caritatis pennis perpetuam salutem. Spectaui, speraui, obtabam : et ecce quem spectaui, non uenit : et quem speraui, non consideraui : quem obtabam, non accipiebam. Frustrata est exspectatio, euacuata est spes. Et utinam pro spe esset praesentia, nunc esset plenum gaudium. Sed, pro dolor, pro exspectata laetitia tristitia inopinata subrepsit. [...] Quid facies modo mens caritate uulnerata? estne aliquod solacium, quod tantum uulnus uel aliquod refocilare ualeat? An spem quasi falsum omnino a te repellis consolatorem? [...] Ecce flamma caritatis totum inardescits pectus, cui si spes collega accessit, feliciter ardet, si ueraciter illi euenit quod optat. Bona est enim spes in absentia; sed melior est amor in praesentia. Nam spes nostra quasi ancora ad caelestis nos trahit patriae soliditatem; sed tunc erit perfecta felicitas, cum plena erit uisio ueri amoris, et uidebitur Deus in gloria sua, qui nunc amatur in pectore nostro. Sed huius exilii peregrinatio patienter feratur, donec optata beatissimae patriae ueniat praesentia. Patientia nobis necessaria est, siue in exspectatione speratae beatitudinis, siue in absentia amicorum carissimorum.
} 
l'espoir loin de toi, en voyant en lui un consolateur mensonger? Voici que la flamme de la charité embrase tout ton être; avec l'espoir à ses côtés, elle brûle avec bonheur, pour peu qu'advienne son souhait. L'espoir est chose bonne dans l'absence; mais mieux vaut encore l'amour dans la présence. Car notre espérance, cette ancre, nous entraîne vers la fraternité de la patrie céleste : c'est alors que le bonheur sera parfait, quand la vision de l'amour véritable sera comblée et que Dieu sera vu dans sa gloire, Lui qui pour l'heure est amour en notre être. Supportons patiemment la longue marche de notre exil ici-bas, jusqu'à ce qu'advienne la présence de la très sainte patrie qui fait tous nos vœux. La patience nous est imposée, dans l'attente de la béatitude espérée, comme dans l'absence d'amis très chers. "

Les citations pauliniennes, devenues motifs alcuiniens, déclinent le plus souvent 2 Co 5,10 (" car nous devrons tous être à découvert devant le tribunal du Christ "), Rm 14, 10 (" tous nous nous présenterons au tribunal de Dieu $^{49}$ "), 1 Co 7, 31 (" car la figure du monde passe ${ }^{50}$ "), 1 Co 3, 8 (" chacun recevra son salaire selon son labeur ${ }^{51}$ ") et Gal 6, 10 (" Ainsi donc pendant que nous en avons le temps travaillons au bien de tous ${ }^{52}$ "). C'est dans ce cadre que s'inscrit l'amitié alcuinienne qui, contrairement à ce qu'on a pu dire n'est pas mystique, mais eschatologique ${ }^{53}$. L'optata praesentia alcuinienne résonne des accents de la parousie paulinienne. De ce mot signifiant présence, Paul livre une interprétation en Rm 1, 11-12; les trois formes que la parousie prend chez Paul, mises en lumière par Régis Burnet, se retrouvent chez Alcuin : la lettre, l'annonce de l'envoi de messagers, représentants de l'épistolier-prédicateur, et la mention de l'intention de voyage ${ }^{54}$. Voici comment Alcuin évoque le thème dans la lettre 279 :

" Je vous ai écrit ces mots d'admonition en père, afin que votre Dilection ait toujours, en ayant cette lettre, ma présence auprès d'elle, comme votre Charité demeure, et pour toujours, solidement ancrée en notre mémoire. Voyez comme il est simple de comprendre au plan de l'amour mutuel ce que le Seigneur en personne dit dans l'Évangile : « Là où deux ou trois sont réunis en mon nom, je suis au milieu d'eux ». Ce qui ne vaut pas seulement, nous semble-t-il, pour la présence physique mais bien plus pour la présence de la sainte charité qui se répand en nos cœurs grâce à l'Esprit Saint ${ }^{55}$. "

49. Alc. Ep. 16, p. 44 et 136, p. 209.

50. Alc. Ep. 16, p. 44.

51. Alc. Ep. 43, p. 88.

52. Alc. Ep. 124, p. 182.

53. Alc. Ep. 220, p. 364 : Credas uelim has litterulas ex caritate dictatas esse ut tu habeas mercedem et frater tuus salutem aeternam.

54. Rm 15, 14-33; Phm 21 sqq. ; 1 Co 4, 14-21; 1 Th 2, 17-3, 13; Ph 2, 19-24; 1 Co 16, 111. R. BURNET, Epîtres et lettres..., p. 100-103.

55. Alc. Ep. 279, p. 436 : Haec scripsi paterna admonitione ut dilectio uestra in hanc cartam meam saepius habeat praesentiam sicut caritas uestra nostrae menti firmiter infixa manebit. Videte quam planum est intellegere in caritate mutua quod in euangelio ipse Dominus dixit : "Ubi sunt duo uel tres congregati in nomine meo, ibi sum in medio eorum. " Non tantum hoc de praesentia corporali dictum putemus sed magis de praesentia sanctae caritatis quae diffusa est per Spiritum sanctum in cordibus nostris. 
La présence revendiquée par Alcuin est alors non seulement élément d'autorité, mais donnée eschatologique qui voit dans la parole écrite et/ou prononcée un élément charismatique.

Ainsi voit-on se modifier la conception antique qui faisait de la lettre un substitut de conversation. La lettre, née de l'absence, nourrie de la distance, et espace de distanciation par les théories qui prennent forme d'elle, installe une présence-parousie d'autant plus complexe qu'elle détermine écriture et lecture de la lettre. Car l'objet lettre, une fois lu par un lecteur, récepteur ou destinataire second, devient parole revivifiée et incarnée dans le corps de qui se met à la lire, démultipliant du même coup les destinataires potentiels ${ }^{56}$.

Dans ces lettres alcuiniennes qui, pour beaucoup, s'ouvrent sur une adresse en Christ disant l'éternité ${ }^{57}$ et s'achèvent sur l'annonce de la venue du Royaume, c'est un au-delà du temps humain qui s'énonce, c'est le Royaume dont l'homme, ici-bas, est séparé qui se dit.

Dans un genre tributaire de codes et conventions, marqué par l'univers patristique, la correspondance alcuinienne témoigne de la richesse de la lettre. Structure mettant en jeu différentes pratiques discursives, la lettre chez Alcuin avance des motifs qui ne relèvent pas de la simple topique mais disent les multiples lois d'énonciations à l'œuvre dans ces textes. La lettre, champ où s'élaborent des modèles d'être et de société, se fait aussi emblème de l'eschaton. Dès lors, la correspondance d'Alcuin n'est pas l'annexe d'une œuvre que constitueraient ses seuls autres écrits, elle est, à part entière, la traduction d'une pensée en marche.

56. E. VALETTE-CAGNAC, La lecture à Rome..., p. 107.

57. Les termes sempiternam salutem (Alc. Ep. 281); perpetuae gloriae salutem (Alc. Ep. 284); in Domino salutem (Alc. Ep. 292); in Christo salutem (Alc. Ep. 300); aeternae beatitudinis salutem (Alc. Ep. 303); perpetuae pacis et prosperitatis in Domino salutem (Alc. Ep. 307); sempiternam caelestis gloriae salutem (Alc. Ep. 308). 


\section{RESUME}

La lettre, souvent utilisée comme document, autorise, en tant que discours, une approche pragmatique au sens linguistique du terme, étudiant l'énonciation afin d'en appréhender le mode de communication. Dans cet article, nous évoquons d'abord le rôle joué par les motifs épistolaires dans la définition de la lettre alcuinienne, puis dans l'émergence d'une identité de l'épistolier, avant de voir, enfin, l'importance des motifs épistolaires, en particulier pauliniens, dans la transformation de la lettre en espace eschatologique. Dans un genre tributaire de codes et conventions, marqué par l'univers patristique, la correspondance alcuinienne témoigne de la richesse du discours épistolaire. La lettre, champ où s'élaborent des modèles d'être et de société, se fait aussi emblème de l'eschaton. Ainsi, la correspondance d'Alcuin, loin d'être l'annexe d'une œuvre que constitueraient ses seuls autres écrits, est, à part entière, la traduction d'une pensée en marche.

\section{ABSTRACT}

Often used as a document, the letter is also a form of discourse allowing an approach which is pragmatic in the linguistic sense of the term, studying enunciation so as to understand its mode of communication. In this article, the author studies the role of epistolary motifs first in the very definition of the Alcuinian letter, and then in the emergence of a specific identity of the letter-writer, before analysing the importance of epistolary motifs, especially Paulinian ones, in the transformation of the letter into a form of eschatological space. In a genre that is heavily codified and conventional, where the universe of the Fathers has left its mark, Alcuin's correspondence testifies to the wealth of epistolary discourse. The letter, a place where models of being and of society are constructed, also becomes an emblem of the eschaton. Thus, far from being a simple appendix to his other, more noble, work, Alcuin's correspondence is an integral part of his thinking on important issues. 
